\title{
On the Function-Behavior-Structure Model for Design
}

\author{
W.J. Zhang ${ }^{1 *}$, Y. Lin $^{2 *}$, and Niraj Sinha ${ }^{1}$ \\ ${ }^{1}$ Department of Mechanical Engineering \\ University of Saskatchewan \\ Saskatoon, SK S7N 5A9 Canada \\ Email: wjz485@mail.usask.ca \\ ${ }^{2}$ Concordia Institute for Information Systems Engineering \\ Concordia University \\ 1455 de Maisonneuve Blvd. West, CB-410-12 \\ Montréal, Québec H3G 1M8 Canada \\ Email: ylin@ciise.concordia.ca \\ * Corresponding author
}

\begin{abstract}
There are many proposals regarding the design model which are related to such notions as function, behavior, and structure. They have created some confusion about and debates on which one should be the most appropriate one. Naturally, a question one may ask is whether they are actually the same thing but with different names or whether they have different scopes of applications for different design problems. In this paper, we evaluate these proposals and attempt to clean up the confusion and comment on the debates. As a result, we are able to conclude a unified and general one and to show how it can be shaped into specific ones, proposed by others in literature, which are applicable to specific design problems.
\end{abstract}

\section{Introduction}

A fair amount of debates can be found on the design model in the context of engineering design from the contemporary design literature. Various proposals on the design model contain to a certain degree notions such as function, behavior, and structure. There are debates on the suitability of these notions to the design model, which have left much confusion. For example, Rosenman and Gero [5] added the notion "purpose" on the top of these three notions. In the work conducted by Umeda et al. [8], the notion "state" was brought into these three notions, and further they appeared to view the state the same way as structure; a functionbehavior-state" modeler was introduced by Umeda et al. [8]. An explanation was given by Hashemian [1] on the difference in the notion of "function" between Pahl and Beitz [3] and Suh [7]; in particular the function in Pahl and Beitz [3] was considered to be input-output oriented, while the function in Suh [7] was considered to be goal oriented. Such an explanation can be commented, which will be discussed later in this paper.

The goal of this paper is to clarify the confusion as mentioned and to conclude a more unified one. The general methodology to achieve this goal is as such; we first present our definition of notions related to the design model, based on the analysis and generalization of many proposals available in the design literature as well as the fundamental understanding of engineering design. We call the set of our defined concepts the FBPSS framework, aiming to take it as a reference model. We then present various schools of thoughts as well as definitions on the design model and comment on them against the reference model (i.e., the FBPSS framework). By doing so, we attempt to show the generality of the FBPSS framework.

This paper will be organized as follows. In Section 2, we present some basic concepts that are needed for subsequent discussions. In Section 3, we present our definition of notions related to function, behavior and structure. Our definition is based on the generalization of those available in the design literature together with our own thoughts [2]. We call the set of notions we defined the FBPSS framework. Section 4 introduces various schools of definitions and evaluates them against the FBPSS framework. Section 5 concludes the paper.

\section{Basic Concepts}

\subsection{Object, artifact, and product}

Object is a thing which is observable by its properties. For example, a bird is an object which is observable by its properties, e.g., flying. A power plant 
is an object which is observable by its properties, e.g., generating power. The object is classified into the natural kind and the man-made kind. The bird falls into the natural kind of object, while the power plant falls into the man-made kind. We call the man-made object the artefact and the natural object either the animal or plant. The human is a kind of animal and is able to create the artefact and alter or modify the animal and plant. When the human does this, he or she applies knowledge (science) and makes use of tool (technology). The process of doing this as such is called engineering. The following phrase is interchangeable: product and artefact; where the product puts more emphasis on manufacturing, making, or producing; i.e., the artefact in this case is produced but not comes to the existence on its own.

In order to have a generalized body of science and technology for engineering, the common natures of all objects are gathered with the name "system". Therefore, the phrase object system (or system for short) means the object which is engineered (or modified when a natural object is concerned). In literature, we often encounter the phrase "system science" and "system engineering". They are applicable to the object as mentioned. It is noted that the state-of-the-art of design theory and methodology today has benefited considerably from such a system's view of the object to be designed.

\subsection{Design and design model}

It is widely agreed that the design of a product system (system for short) is the synthesis of various candidate systems in fulfilling the design requirement. The design requirement includes (1) the need of the system suggested by customers or users and (2) the effort limited to produce the system. The need further has two aspects: (i) the time-insensitive need, and (ii) the time-sensitive need. The (ii) commonly refers to the delivery time of a system. The effort limited is basically about the cost (for producing the system) which can always be converted into the monetary measure. Often, in the design literature and design practice, we encounter the term "function" (of a system); the "function" in this case is a part of the need, specifically the time-insensitive need. A definition of the function will be provided later in this paper.

The candidate systems differ from each other in terms of their geometry, topology, material, and various properties of the material. The design or synthesis aims to choose one among all candidate systems to "best" meet the need with the "minimum" effort.

The above is about the concept of design. The design in its concrete sense implies a process, namely design process. In order to generalize many well- known and specific design processes, one needs a model which is a representation of the process. Ideally, such a model is a formal one - the one based on well defined logics. Such effort has been taken by researchers, such as Zeng and Gu [11].

Another line of development is to provide the ontology of design which is a set of fundamental notions of products / designs and their relationship / constraints. This development has led to the active research related to what is concerned by this paper, namely, the function-behavior-structure framework. Design is a notion which is conceptual; while the design process is practical and follows a sort of logics. In a sense, the design notion offers a meta framework or model for the design process. The term design model refers to a representation of the design notion; while the term design process model is a representation of the design process. In this paper, we concern the design model NOT the design process model. Further, we regard that a more generic design model, in essence, consists of a set of basic concepts and their relationships (ontology) about designed objects.

\section{Generic Definition of the Function, Behavior, and Structure}

The origin of the concept of function, behavior, and structure may go back to the seventies when Rodenacker [4] proposed a design methodology as a guideline for novice designers. In his methodology, a designer first determines the entire function of an entity from the given specification. The function is divided into sub-functions, sub-functions into sub-subfunctions, and so on, until the level where physical behaviors perform such sub-functions. Subsequently, the functional structure is copied to the physical structure. Since then many studies on the function, behavior, and structure concepts in the engineering design community have been conducted, which form several schools. The concept of function and structure is not new to biology, where the cellular behaviors are described using the notion of function and structure. In the artificial intelligence community, the notion of function, behavior, and structure is used for qualitative simulation and fault diagnosis. In the following, we present our definition of several notions, which leads to a design model called the FBPSS framework [2].

\section{Structure and State}

A system has a structure which is a set of entities connected in a meaningful way. The system has a boundary which isolates the system from its environment. These entities are perceived in the form of their states when the system is in operation. States of the entities are thus quantities (numerical or 
categorical) of either physical or chemical domains. The states change with respect to time, which implies the dynamics of an underlying system. In order to express the dynamics of the system in a formal way, the states are expressed by the variables called state variables. In the following discussion, the state variable may be interchangeably used with the state when confusion does not arise. The states are related to one another in various ways because of the constraints imposed on the structure. The constraints come in two forms: the internal and the external. The internal constraints are those related to the connection between entities that form the system at time $t$. The external constraints are those imposed from the environment to the entities of the system. The state variable can be further divided into the independent state variable and the dependent state variable. The independent state (variable) receives the information, energy, material from the outside of the system; while the dependent state (variable) offers information, energy, material to the outside of the system. Figure 1 shows an example of the system, a crank-slider linkage in this case. It is supposed that a powered motion is given to the crank, the motion of the crank is transferred to the coupler, and the motion of the coupler is further transferred to the slider. The slider performs the stroke motion subject to some load. In this example, the motions of the links are represented by variables, i.e., $\theta_{1}, \theta_{2}, S_{3}$. They are the state variable. For a particular system, its state variables and their relationships are determined. The state is to make the structure observable and functional.

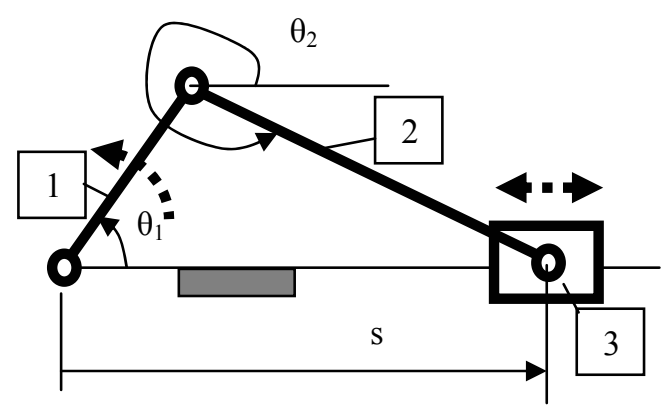

1: crank; 2: coupler; 3: slider

Fig 1. The crank-slider linkage

From a synthesis point of view, not all state variables are useful. For example, in designing a motion converter for changing rotation to translation based on the crank-slider solution concept, the state variable, $\theta_{2}$, is not of a concern. Further, the state and the structure are related such that one state variable can correspond to many structures. For example, the motion converter, as mentioned before, can also be fulfilled by the rack-and-pinion system; see Figure 2 . This has given the evidence that there is a good reason to have two distinct concepts, i.e., the state and the structure.

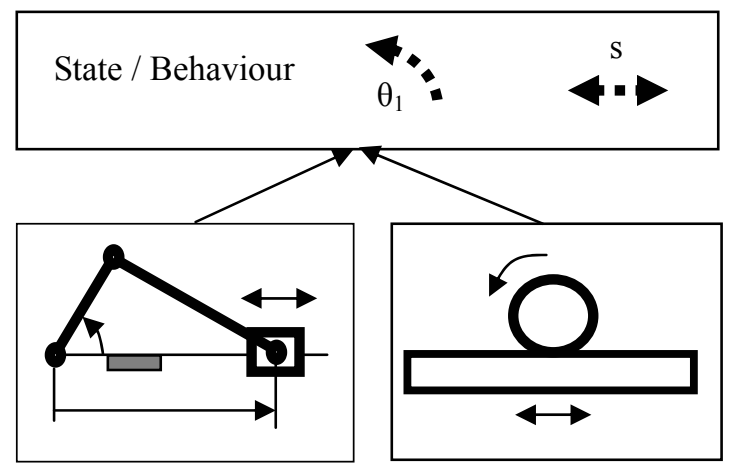

Fig 2. The state versus the structure

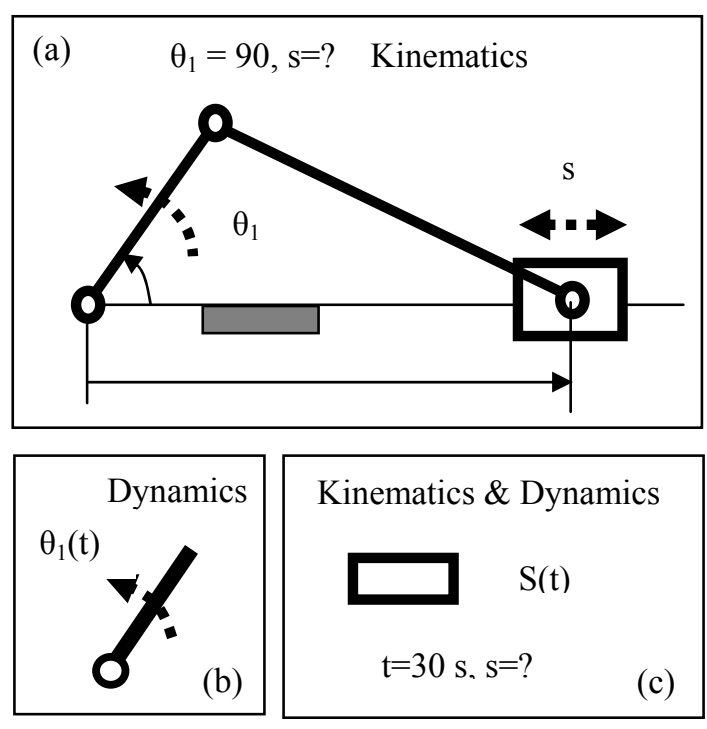

Fig 3. The state versus the structure

\section{Behavior}

The behavior of a system is about the response of the system when it receives stimuli. Since the system (structure) is represented by its state, the stimuli and the response are further represented by the state variable. So, the behavior is the relationship between the independent state variable and the dependent state variable. The kinematic behavior refers to the relation between the dependent state variable and the independent variable; while the dynamic behavior refers to the relation of the independence state with 
respect to time. The system behavior can be (i) the kinematic behavior (Figure 3a), (ii) the dynamic behavior (Figure 3b), and (iii) both the kinematic and dynamic behavior (Figure 3c).

The above definition does imply that the behavior is about the relation between inputs and outputs. Such an input-output relation may not appear apparent for some design situations. For instance, Figure 4 shows a kind of supporter. The usefulness of the supporter system is usually expressed by the phrase 'support load' (P), which sounds more like a statement of function (see later discussions). Yet, for the structure to be capable of supporting the specific load (e.g., $\mathrm{P}=10$ $100 \mathrm{~kg}$ ), the structure should have such a behavior that when there is a force applied at the point $\mathrm{P}$ (state 1), the maximum stress in the structure (state 2) must be less than the allowable stress; here a kind of inputoutput relation (i.e., state $1 \sim$ state 2 ) is implied.

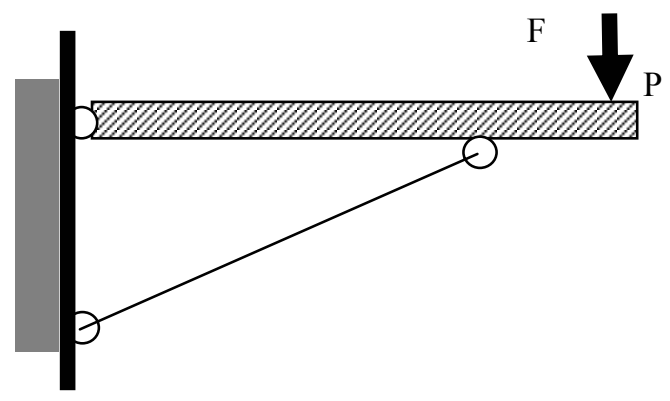

Fig 4. Supporter

\section{Principle}

The principle is the fundamental law with which one can develop a quantitative relation for the state variable. The principle is to govern the behavior as the relationship / constraint among a set of state variables is established based on a particular principle or particular principles. For instance, the Newtonian second law describes the quantitative relation between the force and the acceleration. Another example is the kinematic behavior which is governed by the integrity of an assembly (e.g., the geometry closure of a polygon). The relationship among $\theta_{1}, \theta_{2}$, and $s_{3}$ for the crank-slider linkage system can be established by the geometry closure principle; specifically, we have (for the crank-slider linkage; see Figure 5) as follows:

$$
\overrightarrow{l_{1}}+\vec{l}_{2}=\vec{l}_{3}
$$

where $\vec{l}_{i}(\mathrm{i}=1,2,3)$ are vectors as defined in Figure 5 . Writing (1) into two scalar equations leads to

$$
\begin{aligned}
& l_{1} \cos \theta_{1}+l_{2} \cos \theta_{2}=s_{3} \\
& l_{1} \sin \theta_{1}+l_{2} \sin \theta_{2}=0
\end{aligned}
$$

The above two equations, (2) and (3), are to constrain the state variables, $\theta_{1}, \theta_{2}$, and $s_{3}$ such that to a definite $\theta_{1}$ there will be a definite $s-a$ kind of kinematic behavior with this system.

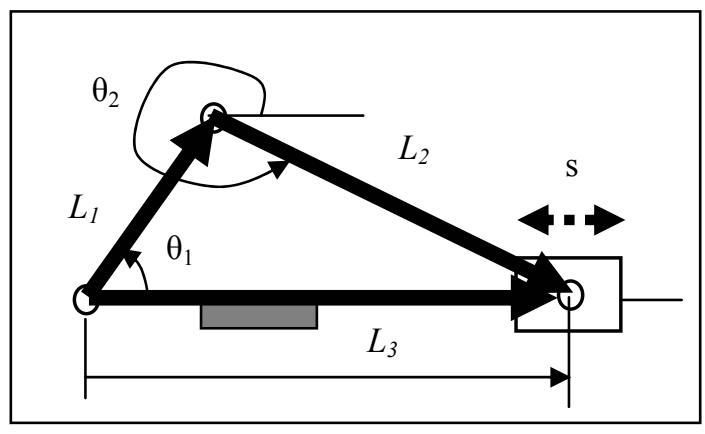

Fig 5. Geometry closure principle

\section{Function}

A system must be useful from its existence, which implies its function. The usefulness of a system is context-sensitive. For instance, the crank-slider mechanism can be used to generate a force on the end of its slider such that the needle attached on the slider can punch the cloth with upper thread (in the context of sawing machine). The crank-slider mechanism can also be used for compressing the gas when its slider is used as a piston in a cylinder where the gas lies.

The two kinds of usefulness of the crank-slider mechanism, as mentioned above, share a common feature or behavior of the crank-slider mechanism, that is, the motion conversion between rotation and translation; particularly in this case, to convert a "full" rotation to a translation with a limited stroke. Subsequently, associated with this motion conversion behavior, the force conversion occurs, i.e., the conversion between moment and force; particularly in this case, to convert a moment on the crank to a force on the slider. The difference in usefulness of the same crank-slider mechanism system lies in the different use contexts for the common behavior of the system. At this point, context one: to compress the gas; while context two: to punch the cloth.

It appears that a function is formed with two steps: The first step achieves a generic role (in the example of the crank-slider, it refers to the motion conversion/force conversion); while the second step achieves a specialized role, depending on what and 
how the system deals with its environment in the crank-slider example (one: the gas; two: the cloth). The environment entity includes the following three types: energy, material, and information. The entities of these three types are processed through systems with different architectures and / or structures. The specialization in function thus depends on the types of these environmental entities. In the first usefulness of the crank-slider mechanism, it deals with the cloth and makes sense of material processing. In the second usefulness of the crank-slider mechanism, it deals with the gas and makes sense of energy conversion (depending on a further application of the compressed gas in the cylinder). The specialization in function also lies in how the generic function is used. For instance, the motion conversion of the crank-slider mechanism may be used for converting translation to rotation; in this case, when the compressed gas in the cylinder involves in the process of generation of heat energy, the mechanism serves as an engine (energy conversion: heat energy to mechanical energy).

The formalization of the function follows the architecture of function discussed above, namely, the generic role and the specialized role. The syntax of the function can be tailored as follows:

\section{Template for function:}

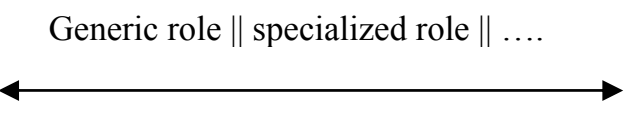

\section{Generalization Specialization}

In the above template, there is an open end in the direction of specialization. This is for giving syntax for getting into a more specific description of the semantics of a function. In the following we give an example to show how to apply this template to give a complete description of a function.

Suppose in the example of compressing the gas we require to straightly compress the gas from $1 \mathrm{~Pa}$ to $3 \mathrm{~Pa}$ by revolving an object. The function in this case can be expressed as follows:

Generic role 1: motion conversion; Generic role 2: force conversion; Generic role 3: pressure generation;

Specialized role 1: rotation to translation (rotation: full; translation: reciprocating);

Specialized role 2: torque to force;

Specialized role 3: gas from $1 \mathrm{~Pa}$ to $3 \mathrm{~Pa}$.

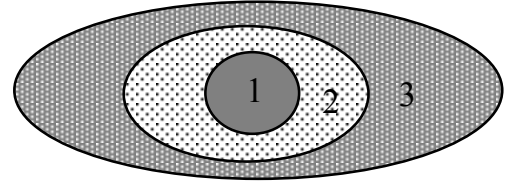

2 is a context of $1 ; 3$ is a context of 2

Fig 6. Relativity principle in the context

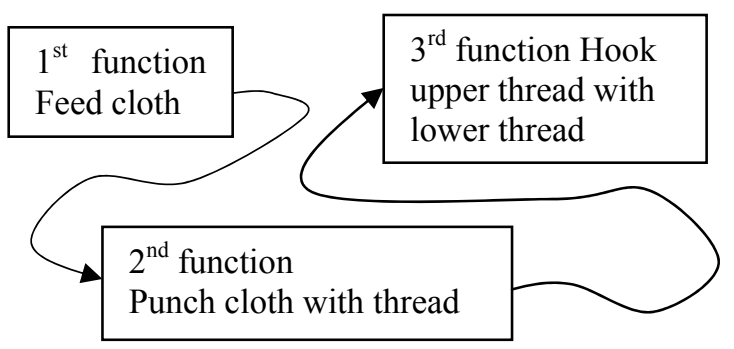

Fig 7. Decomposition of the sewing machine

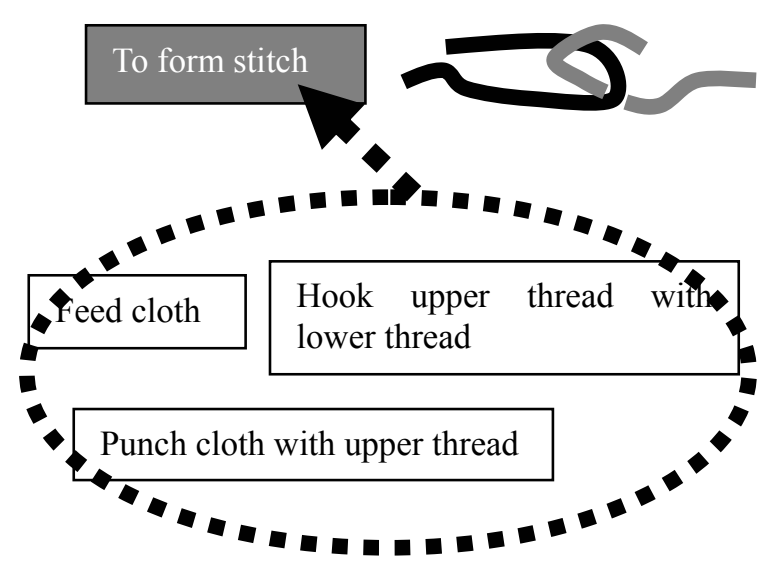

Fig 8. Context relativity - form stitch

It is further noted that the subjectivity of the function is related to its "direct" context which indicates the rationale or purpose for a particular behavior to be associated with it. Furthermore, this context is relative in development throughout the ladder of components, subsystems, and system; see Figure 6 . The context can be expressed by the precondition and the post-condition for a particular behavior to "fill" in. For instance, the function of a sewing machine can be decomposed into (partially): feed the cloth, punch the cloth with upper thread, and hook the upper thread with lower thread [13]. The second function is fulfilled by the crank-slider linkage 
with its pre-condition (the first function) and its postcondition (the third function); see Figure 7. The function of the subsystem which consists of these three functions is to form a stitch chain (see Figure 8).
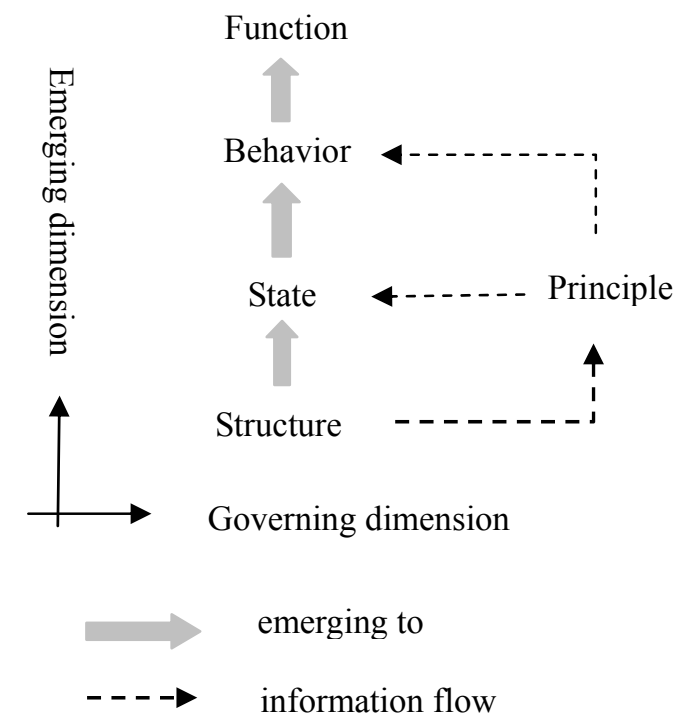

Fig 9. Relationship among various concepts

\section{Relationship among these concepts}

A set of states may correspond to more than one structure (see Figure 2). So the relationship between the state (a set of states in this case) and the structure is a one-to-many relationship. The behavior is an aggregate of a set of states, while a particular aggregation depends on the underlying structure and the principle (see Figure 9). The relationship between the behavior and the function is a many-to-many relationship; i.e., a particular behavior may be used for different functions under different contexts, while a particular function may be achievable by more than one behavior. There is no any principle that tells why a particular behavior can serve for a particular function. Here, we imply the subjectivity aspect of a function. While rationale exist for whether a particular behavior could achieve a particular generic function; for example, whether the crank-slider mechanism can achieve the motion conversion function (a generic function), from rotation to translation, depends on the geometry of the mechanism (i.e., $\mathrm{L}_{2}$ must be greater than $L_{1}$ in Figure 1). This implies that the objectivity does exist between the behavior and the generic function concepts.

\section{Further remarks}

Remark 1: A system is usually decomposed into sub- systems, and components. The FBPSS model applies to all these objects. A design of machine systems is generally divided into three design stages: the conceptual design, the embodiment design, and the detailed design. The FBPSS framework is applicable to all these stages. Figure 10 shows a triad of the FBPSS, the system decomposition, and the general design stage.

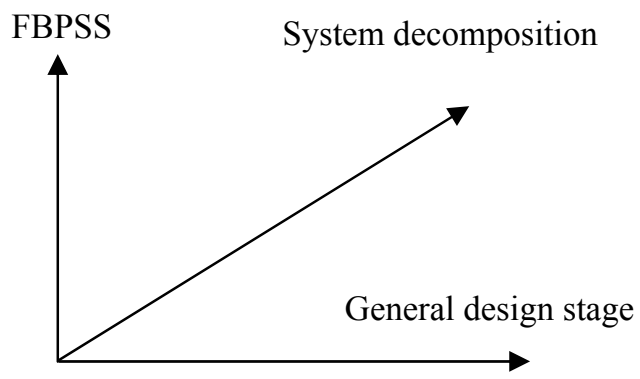

Fig 10. The design model triad

Remark 2: A system, subsystem, or component may serve for different functions. For instance, in the example of the crank-slider mechanism, it serves for both motion conversion and force conversion. In this case, the FBPSS model applies to all these functions.

Remark 3: There are two reasons, any of which is to account for the distinction of those concepts, i.e., function, behavior, principle, state, and structure. The first reason is that there is a one-to-many or many-tomany relationship related to any two of these concepts. For instance, there is a one-to-many relationship between the function and the structure concepts; i.e., both the crank-slider and rack-and-pinion structures could play a role as motion conversion and force conversion. That said, the two concepts, function and structure, must be distinct. The second reason is that there is an aggregation relationship related to any two of these concepts. For instance, a behavior presents an aggregated relation with a set of states; in the example of the crank-slider mechanism it is the set of states, $\theta_{1}$, $\theta_{2}, S_{3}$, that comes together under one governing principle to form the behavior that when the crank rotates to an angle the slider moves a certain amount.

\section{Different Schools of the Design Model}

Having had the FBPSS framework as a reference we now comment on various proposals of the design model that are related to the notion of function, behavior, and structure.

School 1: Australian school of the design model. 
The following concepts were defined in this school [5]:

Purpose: the reason why an artefact exists or why it is what it is, what it is intended for;

Function: the thing an artefact performs;

Behavior: the manner in which an artefact acts under specified conditions; and

Structure: what constitutes an artefact (or defines its constitution).

They further explained that a purpose is an intended function; in other words, a purpose is a subset of functions as an artefact can have many functions. For example, a motor car carries things, exhausts fumes, makes noises, etc. The last two did not fall into the domain of purpose. The following is a comparison between their definitions of concepts and the FBPSS framework:

\begin{tabular}{ll}
\hline School 1 & FBPSS \\
\hline Purpose & Specialized function \\
Function & Generic function \\
Behavior & Behavior \\
Structure & Structure \\
\hline
\end{tabular}

From this comparison, one can see that they do not explicitly identify the state. We believe that to have a distinct concept, state, from the behavior and structure concepts, is more in line with the design logics. For the example of the crank-slider mechanism for the purpose of punching cloth with upper thread, the logics of design or synthesis process is:

\section{Punch cloth with upper thread -> ? Generic function (motion conversion: rotation to translation) -> ? States (one state for angular displacement and one state for translational displacement) $->$ ? Structure (crank-slider or rack-and-pinion).}

In the above, the notation "?" stands for "what is about".

School 2: Japanese school of the design model. The related concepts defined by this school [8,9] are function, behavior, state, and aspect. Specifically, this school defines a function as a description of behavior abstracted by human through recognition of the behavior in order to utilize the behavior as sequential state transitions along time. In this school, the function is considered as a subjective matter, which corresponds to the specialized function in FBPSS. They recognized the relationship between the function and behavior (in their definition) as a many-to-many relation. They have not presented any relationship between the state and the structure, they have given the definitions for the state and the structure, respectively [9]. Their model does not recognize the fact there is still some objectivity between the behavior and the function, which is important to develop a systematic process to conduct design. Note that the subjectivity matter usually implies a certain kind of ad-hoc process. Furthermore, the function in school 2 is the same as the purpose in school 1. The concept of aspect in their design model is close to that described in Remark 2 in the previous discussion. It is also noted that their definition of the behavior is exclusively related to the time, which may exclude the kinematic behavior in the example of the crank-slider linkage (Figure 1) as well as the stress and strain behavior in the example of the supporter (Figure 4).

School 3: American school of the design model. The first definition of function, behavior, and structure in the United States was proposed by De Kleer [1984]. According to him, structure is 'what the device is,' behavior is 'what the device does,' and function is 'what the device is for.'

The definition in this school is the same as those in school 2 except that in this school state is omitted. Furthermore, Suh [7]'s definition of the function concept is the same as that in school 2.

School 4: European school of the design model. This school puts forward the following definitions:

Function: is the usefulness of the behavior, perceived by the human user;

Behavior: is a description of the system in terms of its allowable states, the system's variables, and how those variables are related;

Structure: is how the behavior is realized.

A popular approach in this school to represent the function is to have a pattern called "input-output". The function is viewed with this pattern as fulfilling the transfer of an input with a certain kind to an output with a certain kind. The types of the kind are material, energy, and information. This view was criticized by other schools, e.g., school 2 and Hashemian [1] as not being able to represent the function with the generic pattern called "verb+noun" (or called "goal-oriented" pattern) (e.g., to support load, punch cloth, etc.). We do not entirely agree on this criticism. We believe that from somewhat an absolute sense, a system does perform a certain input and output transfer, which is the nature of the behavior (stimuli-response). However, in the design practice, sometimes, one may feel more 
conformable with the input-output pattern; while in another occasion, the goal-oriented pattern is more natural. For instance, 'transfer a rotation to a translation' appear to be more natural with the inputoutput pattern, while 'support load' may appear to be more natural with the goal-oriented pattern. Further, whether it should be a goal-oriented pattern or an input-output pattern for a particular function may depend on the level of details in description. For instance, one can say 'convert motion' at a certain level of design; one must give further detailed information of this motion conversion, specifically rotation to translation at a more detailed design level.

In summary, we note that none of these schools recognizes the relationship between the state and the structure in FBPSS. This relationship is important to have somewhat a complete design process. None of these schools explicitly includes the principle, which again leads to incompleteness in the description of a design. Note that in the design of computer-user interface, the principle concept is crucial to fault diagnosis in an unknown situation [2].

The argument on the function concept, specifically on the goal-oriented pattern or input-output pattern, reflects the inflexibility in those design models in the sense that only one specific view holds with them, respectively. The problem of inflexibility in representing concept is now identified in the design model; while the similar problem, the inflexibility in representing semantics (unable to represent different views on the same piece of data) in database modeling and design, have been recognized long before $[6,12]$.

\section{Conclusions}

The FBPSSS framework consists of the following distinct concepts, i.e., the function, behavior, principle, state, and structure. The function concept is further divided into the generic function and the specialized function. Their relationships are developed along two dimensions, namely the emerging dimension and the governing dimension; see Figure 9. The FBPSS framework is orthogonal to the system decomposition concept and the general design state concept, respectively; see Figure 10. It is noted that for a particular design problem not all these concepts may be applied. For instance, in the design of a humancomputer interface for the website for the purpose of information and knowledge searching and acquisition, only the function and the structure concepts are needed [10]. The FBPSS framework is perhaps more useful to the conceptual design stage but not to the embodiment and detailed design stages at which the synthesis problem can readily be represented by an optimization problem.

Acknowledgement: This research has been partially supported by NSERC through a Discovery Grant to the first and second authors.

\section{References}

[1] M. Hashemian, Design for adaptability, Ph. D. thesis, Department of Mechanical Engineering, University of Saskatchewan, 2005.

[2] Y. Lin and W.J. Zhang, "Towards a novel interface design framework: function-behavior-state paradigm," International Journal of Human Computer Studies, Vol. 61, 3, 2003, pp. 259-297.

[3] G. Pahl and W. Beitz, Engineering design: a systematic approach, Springer-Verlag, Berin, 1988.

[4] W. Rodenacker, Methodisches Konstruieren, SpringerVerlag, Berlin, 1971.

[5] M.A. Rosenman and J.S. Gero, "CAD modeling in multidisciplinary design domains," In Artificial Intelligence in Structural Engineering (I. Smith, Ed.), Springer, Berlin, 335-347, 1998.

[6] J.M. Smith and D.C.P. Smith, 1978, "Principles of database conceptual design," Proc. NYU Symp. on Database Design, 35-49, 1978.

[7] N.P. Suh, The Principles of Design, Oxford University Press, Oxford, UK, 1990.

[8] Y. Umeda, H. Takeda, T. Tomiyama, and H. Yoshioka, "Function, behavior, and structure," In Applications of Artificial Intelligence in Engineering, V, (Gero, J.S., Ed.), Computational Mechanics Publications and Springer-Verlag, 177-193, 1990.

[9] Y. Umeda, et al., "Supporting conceptual design based on the function-behavior-state modeler," Artificial Intelligence for Engineering Design, Analysis and Manufacturing, 10, 1996, 275-288.

[10] Y.W. Wang, Towards a novel approach for website interface design: FBS-WEB approach, M. Sc thesis, Concordia Institute for Information Syste ms Engineering, 2005.

[11] Y. Zeng and P. Gu, "A science-based approach to product design theory. Part I: formulation and formalization of design process," Robotics and Computer Integrated Manufacturing, Vol. 15, No. 4, 1991, pp. 331-339.

[12] W.J. Zhang and K. van der Werff, "A critique of conceptual data modeling notions relative to the machine design domain," Proc. of 1994 ASME Engineering Database Symposium'94, Sept. 11-14, Minneapolis, USA, 59-66, 1994.

[13] W.J. Zhang et al., "Working principles and mechanism analysis of Industrial sewing machine (in Chinese)," Publisher: Shanghai Industrial Sewing Machine \& Equipment Research Institution, Shanghai (P.M. Li, Ed.), 1990. 